\title{
LASER SPOT DETECTION AND CHARACTERISTIC ANALYSIS IN PLASMA INTERACTION SIMULATION
}

\author{
Zhiwei Ai, Yi Cao, Huawei Wang and Li Xiao \\ Institute of Applied Physics and Computational Mathematics, Beijing 100094, China \\ CAEP Software Center for High Performance Numerical Simulation, Beijing 100088, China
}

\begin{abstract}
The analysis of beam divergence mechanism during laser plasma propagation is one of the hot topics research. Among them, laser spot detection and characteristic analysis is one of the important indicators to evaluate the energy distribution of the exit surface of the propagation system. The channel environmental factors during the plasma laser transmission are complex and changeable. This scene will cause distortion of the laser spot, making it difficult to achieve spot detection and precise analysis of characteristics. This paper presents a novel visual analysis method to study the beam divergence mechanism. First, the dynamic threshold segmentation method is used to analyze the numerical simulation data to generate a laser spot image. Second, the use of image edge detection technology to achieve spot contour drawing based on image pixels. The above two key technologies have been applied to the accurately positioning of the spot contour and the analysis of the spatial distribution characteristics of the spot. Experiments show that this technique is helpful to verify the correctness of the physical model of the laser plasma interaction simulation program, and is also effective.
\end{abstract}

\section{KEYWORDS}

Laser Profile, Image Processing, Circle Fitting

\section{INTRODUCTION}

The important of the spatial characteristic distribution and characteristic analysis of the laser spot is reflected in the correct recognition of the physical model and data modeling. Diagnosing data quickly is one of the difficulties faced by data analysis in laser-plasma interaction simulation. In the analysis of the beam divergence mechanism when the bundled light propagates in the plasma, the visual analysis of the spatial distribution of the laser spot profile is a hot spot of concern. The channel transmission environment during plasma laser propagation has diversity and complexity. In the process of plasma laser propagation, laser spot drift, intensity flicker, dispersion, distortion and other phenomena may cause the energy of the laser receiving end to diverge. In severe cases, the laser energy propagation is interrupted. Various factors cause distortion of the laser spot, making it difficult to accurately locate the spot profile. In the process of space laser propagation, the precise positioning of the spot profile will greatly affect the power of the laser transmission exit surface, which restricts the analysis of the beam divergence mechanism when the beam is propagated in the plasma.

According to the different positioning principles of traditional laser spot contour positioning, the algorithm can be divided into two types, including direct positioning method and indirect positioning method. Direct positioning method is to use discrete numerical calculation method to directly detect the spot contour on the laser spot image, such as threshold segmentation method (Shen et al., 2012) and edge detection method (Orujov et al., 2020). The indirect positioning rule is to calculate the centroid and beam waist parameters of the laser spot, and indirectly calculate the spot profile quickly. Common centroid algorithms include gray center of gravity method (Jeppe et al., 2017), ellipse fitting method (Kurt el al., 2012), Gaussian fitting method (Wu et al., 2017), etc. Figure 1a shows the energy distribution provided by the spatial distribution of the flat-top beam. Among them, the traditional technology only calculates the spot light centroid, beam width and other characteristic parameters to analyze the spot profile change, but it cannot calculate the fitted circle radius of the spot profile. The accuracy of the centroid calculation of this method will greatly affect the detection error of the spot contour. The paper proposes a novel solution. This method 
adopts a laser spot radius solving algorithm based on circle fitting and an image-based precise positioning and drawing algorithm for spot contours, to realize the visual analysis of the beam divergence mechanism during the propagation of concentrated light in plasma. As shown in Figure 1b, this method uses a physical field-based threshold segmentation method to describe the characteristic spatial distribution of the light spot. The white dotted line describes the outline of the laser spot, and calculates the energy deposition equivalent area of the spot to obtain the fitted circle radius of the spot. Experiments show that the algorithm can accurately locate the spot contour. At the same time, this method is suitable for the spot fitting circle radius of the batch mode of time series data. Therefore, this method is helpful to verify the correctness of the physical model of the laser plasma interaction simulation program. This method is helpful to verify the correctness of the physical model of the laser plasma interaction simulation program, and is also effective.

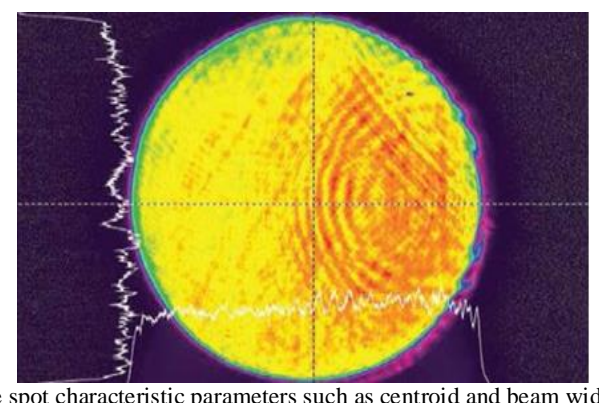

(a) The spot characteristic parameters such as centroid and beam width

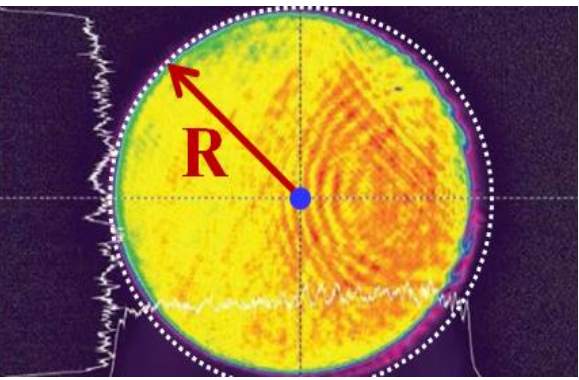

(b)Accurately locate the spot profile and calculate the radius of fitting circle

Figure 1. Laser spot contour positioning algorithm based on threshold segmentation

The remainder of this paper is structured as follows. Chapter 2 introduces commonly used detection techniques for laser spots. Chapter 3 introduces the two key technologies used to achieve laser spot detection. First, use dynamic threshold segmentation to detect and identify laser spots. Second, the image-based edge detection technology is used to realize the spot contour positioning and drawing algorithm, and the spot contour is drawn. Chapter 4 introduces the analysis of the spot characteristics in the laser plasma interaction simulation program.

\section{RELATED WORK}

At present, whether it is from the hardware or software, many have spent a lot of energy on the positioning research of the laser spot contour. Lambert et al. (1995) analyzed the factors that caused the laser beam distortion caused by atmospheric laser communication in detail from the aspects of space channels, transceiver devices and laser carrier. Jason et al. (2008) in the American Aerospace Corporation built a laser communication hardware and software test platform, and have always insisted on combining hardware and software to seek to design the best algorithm. From a morphological point of view, Singh et al. (2008) used Gaussian fitting curves to locate the spot centroid at the accuracy of sub-pixels. Yu (2006) proposed a spot center correction method for aberration data calculated by Zernike polynomials. Liu (2014) used Houng transform to improve the algorithm of laser spot center positioning accuracy. Wang et al. (2011) and Liu et al. (2011) introduced a curve-fitting sub-pixel positioning algorithm for the center of gravity. Tang et al. (2009) used Gaussian cumulative distribution fitting method 5 and Li et al. (2013), Wang et al. (2013), Zhao et al. (2014) and Qin et al. (2006) designed and implemented the least squares circle fitting in parallel to determine the spot center position and radius. Wang et al. (2007) started from the modulation method, modulating the communication light into circularly polarized light, reducing the introduction of noise in communication, and then using dynamic threshold segmentation to detect the spot profile. Mu et al. (2011) used the information distribution between time series data to control the error to a very small range, and determine the spot profile according to the ellipse fitting method.

With the continuous improvement of parallel computing power, the scale of computing grid of simulation program is also increasing. Taking the laser plasma interaction simulation program as an example, the grid size is close to 10 billion grid cells. Laser plasma interaction involves coupling multiple temporal- and spatial-scale nonlinear processes with very important physical phenomena. To study the generation and development of laser filament instability in the black cavity, a propagation model of the spatial beam uniform beam was established. The Lap3D simulation program was used to simulate large-scale filament formation 
under the physical conditions of the black cavity (Hu et al., 2015). The simulation program provide the results corresponding to the laser, initial intensity $\mathrm{A}_{0}=2 \times 10^{15} \mathrm{~W} \mathrm{~cm}^{-2}$, wavelength $\lambda=1.05 \mu \mathrm{m}$, and irradiated neutral plasma with uniform initial electron density and temperature. The electric field amplitude of a light beam is considered a Gaussian function with $\mathrm{z}=0$. Volume rendering with laser intensity of $56.6 \mathrm{ps}$ (Figure 2) reveals significant filamentation. In this example, simulating a physical time of 56.6 ps requires 16,384 CPUs with $1024 \times 1024 \times 2048$ cells (approximately 21 billion cells). The total time-varying data of the analog output of the entire process of 104 steps is $1.74 \mathrm{~TB}$, and the parallel visualization is completed by using $128 \mathrm{CPU}$ cores.

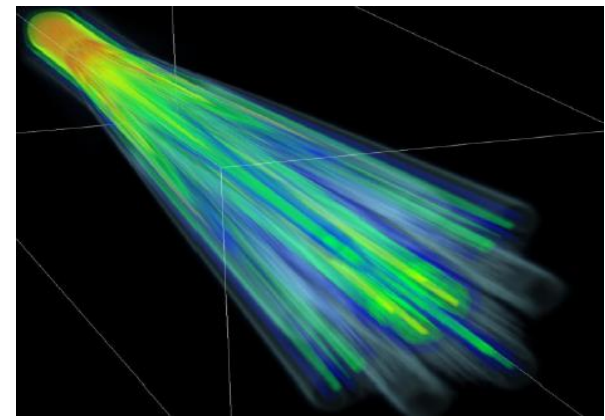

Figure 2. The volume plot of the laser intensity in 3D

Spot detection and characteristic analysis during laser transmission in plasma are challenging. Most of the traditional methods are image data processing, which cannot guarantee the spot analysis of the complete physical field. This paper proposes a method of spot detection and characteristic analysis based on numerical and image data, which can realize spot data analysis of any laser propagation surface. Therefore, this method can ensure the completeness and correctness of data analysis.

\section{KEY TECHNOLOGY}

This paper presents a novel visual analysis method to study the beam divergence mechanism. Firstly, the threshold-based segmentation method is used to describe the spatial distribution of the laser spot, and the laser spot image is generated. Secondly, the use of image edge detection technology to achieve point contour drawing based on image pixels.

\subsection{Description of Spot Spatial Distribution Based on Threshold Segmentation}

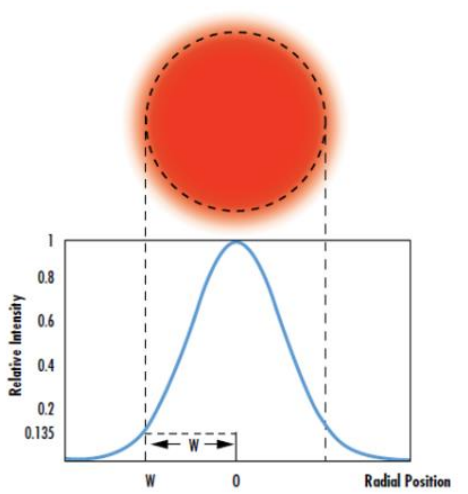

Figure 3. A Gaussian beam's waist is defined as the position where irradiance is its maximum of $1 / \mathrm{e}^{2}(13.5 \%)$

Many laser optics applications assume that the laser beam is a Gaussian beam, and its irradiance distribution conforms to the ideal Gaussian distribution (Lu, 2003). In fact, all actual laser beams will deviate from the ideal Gaussian behavior. The $\mathrm{M}$ factor (also known as the beam quality factor) compares the performance of a real laser beam and a diffraction-limited Gaussian beam. The Gaussian irradiance distribution is 
symmetrical about the center of the beam, and decreases as the distance between the center of the beam along the direction of beam spread increases, as shown in Figure 3.

This distribution is described by Equation $1 . I_{0}$ is the peak irradiance at the center of the beam, $r$ is the radial distance from the axis, $w(z)$ is the radius of the laser beam, $z$ is the wave front, and $P$ is the total power of the beam.

$$
I(r)=I_{0} \exp \left(\frac{-2 r^{2}}{w(z)^{2}}\right)=\frac{2 P}{\pi w(z)^{2}} \exp \left(\frac{-2 r^{2}}{w(z)^{2}}\right)
$$

For Gaussian mode TEM (Transverse Electromagnetic Modes), the beam width defined by the second-order moment is consistent with the beam width defined by the intensity reduction to $1 / \mathrm{e}^{2}$. The beam width defined by this method is very sensitive to the interference of high spatial frequencies, and there is always some base noise in the actual measurement process. Therefore, in this paper, another method is used to select appropriate isobaric lines to divide the power density distribution surface. The cutting plane should be selected so that the cut beam area contains $86.5 \%$ of the total power. At this time, the definition equation for calculating the cutting plane is Equation 2. In the formula, $P_{r c}$ is the power within the beam radius, and $P$ is the total power.

$$
P_{r c}=\left(1-e^{-2}\right) P=0.865 P
$$

In the laser-plasma interaction simulation program, the incident laser beam is mostly Gaussian beam. Based on the Gaussian spatial distribution characteristics, the algorithm proposes a threshold method based on accumulated energy deposition to describe the spatial distribution characteristics of light spots. Specifically, a weight-based histogram of physics field value distribution is used to count the occupancy of the physics field segment field value and total energy value one by one. Discrete the range of the physical field value into 1024 uniform eigenvalue intervals, and calculate the energy corresponding to each eigenvalue interval (energy $=$ eigenvalue value $*$ the number of cells in this eigenvalue). Starting from the smallest feature interval, the energy of each feature interval is accumulated in sequence, and the energy accumulation of the segmented feature interval is calculated. When the ratio of the energy accumulation of the segmented feature interval to the total energy value approaches $86.5 \%$, it is determined that the maximum physical field value in the segmented feature interval is the laser contour segmentation threshold. The segmentation threshold is used as the screening criterion to distinguish the spot range of the physical field data and calculate the spot area. The laser beam also exhibits a normal distribution characteristic at the exit surface, so the fitting circle calculation is used to derive the fitting circle radius of the laser in the plasma propagation process. Figure 4a shows the spatial distribution of laser energy on the exit surface of a calculation model when the number of iterations is 49680; through the histogram of field value distribution based on the laser energy weight, the separation threshold of the laser profile is calculated to be 1.319 . Figure $4 \mathrm{~b}$ shows the grid cells with energy value higher than 1.319 are retained, and the spatial distribution of laser spot is drawn.

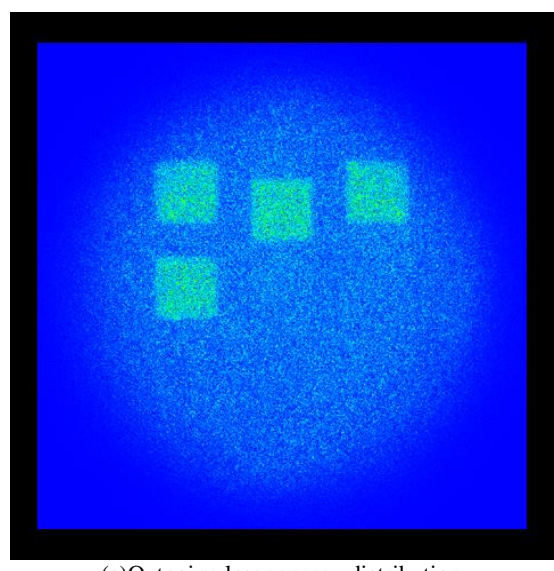

(a)Outgoing laser energy distribution

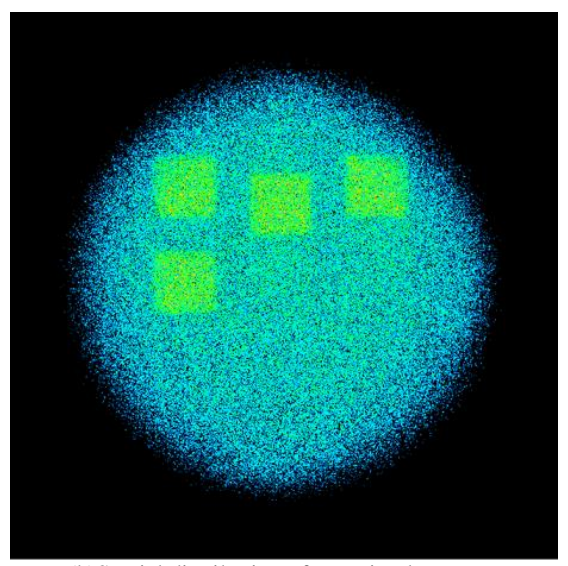

(b)Spatial distribution of outgoing laser spot

Figure 4. A model calculates the spatial distribution of laser spot energy when the number of iterations is 49680 


\subsection{Algorithm for Spot Contour Positioning and Drawing Based on Image Pixels}

The spot profile is a curve connected by continuous points (connected to the boundary), with the same variable value, color value or gray scale. As shown in Figure 5, the contour of the laser spot is obtained by directly using the contour drawing method of the threshold. Since the laser spot is the result of energy deposition, the contour drawing method based on physical values is simply adopted, and the outline of the laser spot cannot be depicted, as shown in the left figure. It can be observed by zooming in on the red local area, the contour distribution presents a locally closed space, and the edge of the laser spot cannot be continuous, as shown in the right part of Figure 5. Therefore, the contour drawing based on physical values cannot describe the outline of the laser spot and cannot meet the needs of data analysis.

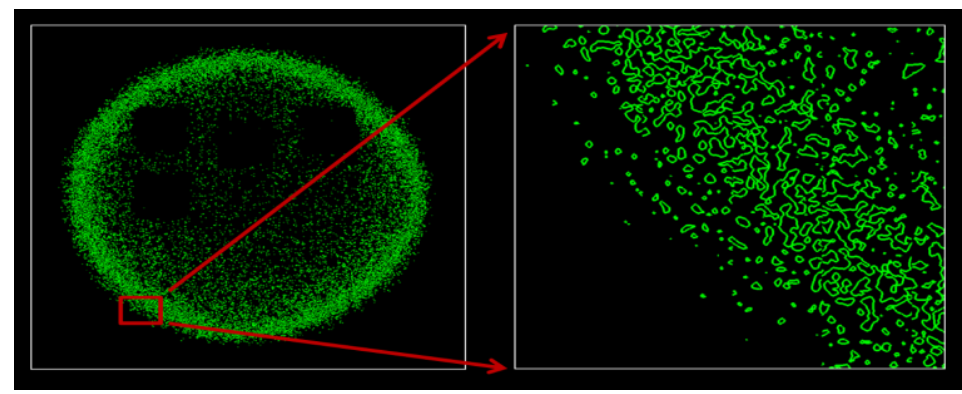

Figure 5. Drawing of threshold contours based on physical quantities

The image-based spot contour drawing method is an algorithm that directly separates the target from the background of the laser spot image. The essence is to find a certain separation value through a series of calculations. With this value as the boundary point, all pixels larger than the separation value are divided into one area, which is generally called the target; all pixels smaller than the separation value are divided into one area, which is generally called the background. In this process, the separation value is called the threshold, and the accuracy of the threshold will determine whether the target can be correctly separated, that is, the spot contour. The input object of the spot pixel positioning and drawing algorithm based on image pixels is the image pixel data of the laser spot. The algorithm flow includes: data filtering; threshold value processing, binary image; laser profile positioning, that is, on a black background, the edge of a white object is the spot contour. The work flow chart is shown in Figure 6. In the first step, read the color fill map of the spatial distribution of the laser spot, the image is a color map with RGBA signal channels. In the second step, the pixel values in the color fill map are subjected to median smoothing filtering to eliminate the influence of interference noise signals. In the steps 3 , the image pixels are normalized to a single pixel value according to RGBA, to achieve the grayscale of the color image. In the step 4, based on the weighted image gray value histogram probability statistics, calculates the image segmentation threshold, according to the image segmentation threshold, and generates a black-and-white binary image of the laser spot. In the step 5, according to the binary black-and-white image, extracts continuous white points and connects them into a line to form a spot contour edge. The last step draws a color fill map of the field amount distribution and the spot contour edge by coupling to obtain the final laser spot profile.

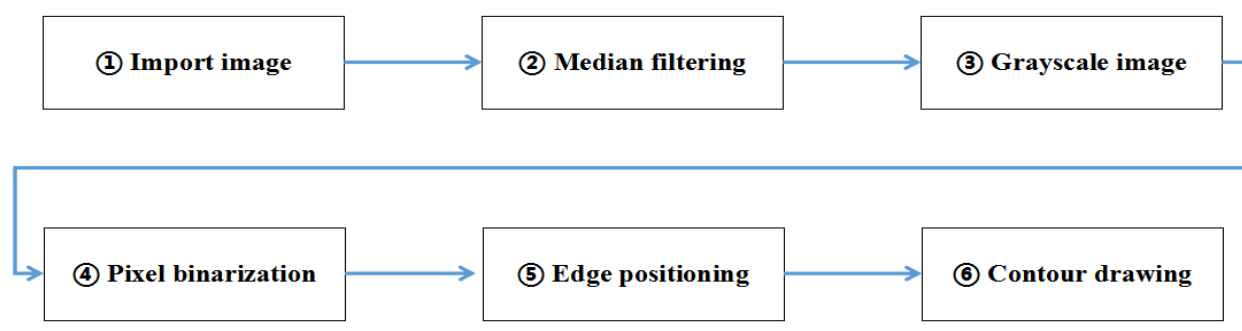

Figure 6. Flowchart of Algorithm for Drawing and Locating Spot Contour Based on Image Pixels

As shown in Figure 7, a schematic diagram of image processing at various stages of the image-based spot contour drawing method is introduced. Figure 7a shows the spatial distribution of spot energy based on circle fitting; Figure 7b shows the gray map of spot energy distribution; Figure 7c shows the gray map after the median filter processing, through the median filter; Figure $7 \mathrm{~d}$ shows the binary image of the smoothed image, 
and the spatial area of the light spot is distinguished by black and white; Figure 7e shows the outline of the white spot area; Figure $7 \mathrm{f}$ shows the effect drawing of the coupling of the spatial distribution of the original light spot energy and the light spot contour.

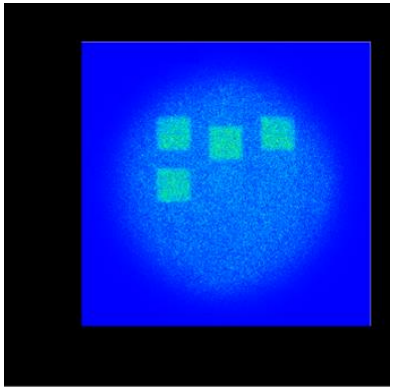

(a)Original pseudo-color image

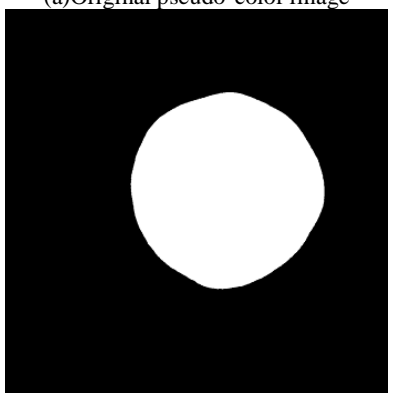

(d)Black and white binary map

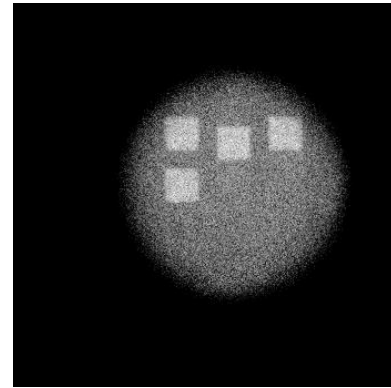

(b)Grayscale image

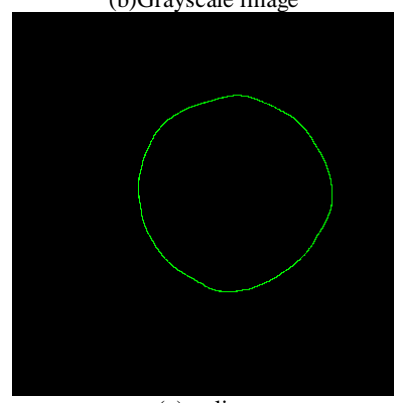

(e)outline

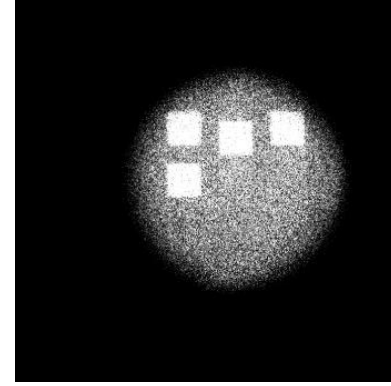

(c)Median filtering

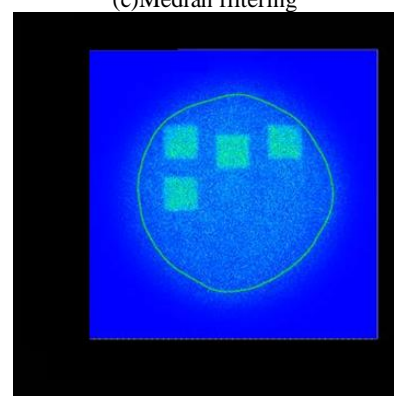

(f)Spot coupling profile

Figure 7. Schematic diagram of image processing in various stages of image-based spot contour drawing method

\section{SPOT CHARACTERISTIC ANALYSIS}

(a)The transverse spatial spectral distribution of the incident surface of four laser beam with polarization direction $\mathrm{X}$

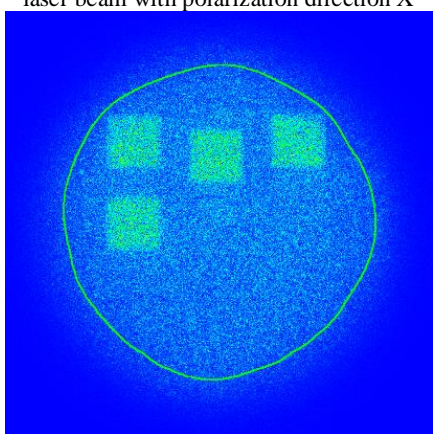

(c) iteration step X-direction polarized laser beam exit surface lateral spatia spectral distribution

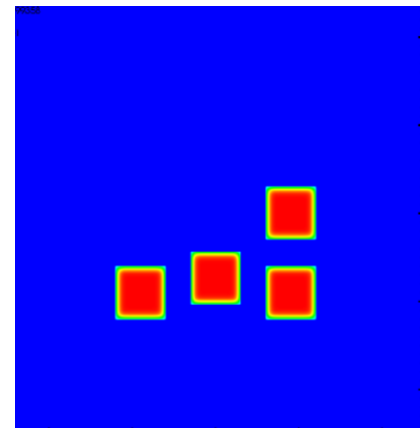

(b)The transverse spatial spectral distribution of the incident surface of four laser beam with polarization direction $\mathrm{Y}$

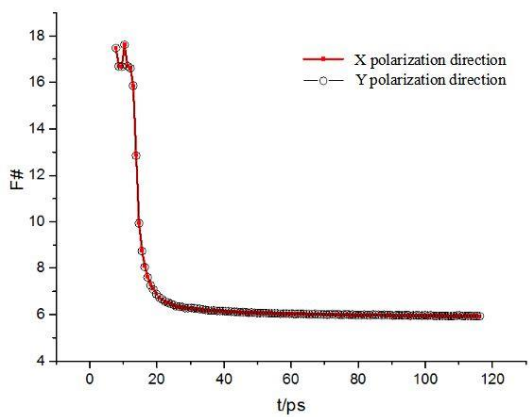

(d)The radius of the fitted circle on the exit surface of the X / Y polarization direction changes with time

Figure 8 .Visual analysis of beam divergence as the beam of light propagates in the plasma 
Spot characteristic analysis is a mean to verify the correctness of the simulation results of the laser-plasma interaction program and to discover important physical phenomena, and has very important practical application value. As shown in Figure 8, when the 8 laser beams are divided into two polarization directions and enter the plasma, the variation law of the circle radius of the beam divergence fit with time when the beam propagates in the plasma is studied. Figure $8 \mathrm{a}$ and Figure $8 \mathrm{~b}$ are the transverse spatial spectral distribution of the incident surface of the four laser beams with polarization directions in the $\mathrm{X}$ and $\mathrm{Y}$ directions incident on the plasma. The red square area is the spatial distribution of the incident light; Figure $8 \mathrm{c}$ shows the spatial distribution of the X-direction polarized laser exit surface when the iteration step number of the simulation program is 46000 , where the green outline is the spatial range of the laser power deposition distribution energy of $86.5 \%$; Figure $8 \mathrm{~d}$ shows the variation of the fitted circle radius on the exit surface with time. The $\mathrm{X}$ direction coordinate is the simulated physical time, and the $\mathrm{Y}$ direction coordinate $\mathrm{F}$ is the derivative of the fitted circle radius. When the four laser beams propagate in the plasma, the beam diverges and reduces the radius of the fitted circle. The experimental results are in line with physical expectations.

Figure 9 shows a time sequence diagram of the lateral spatial spot profile of the X-direction polarized laser beam exit surface at different iteration steps of a calculation model. It can be seen that in the initial stage of laser propagation, the spot profile distribution is irregular. With the evolution of time, the outline of the spot is gradually regular, and the radius of the spot remains basically unchanged.
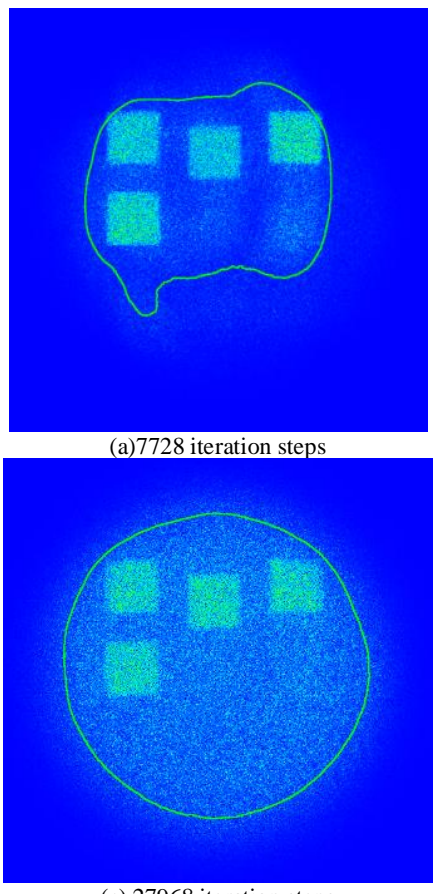

(c) 27968 iteration steps

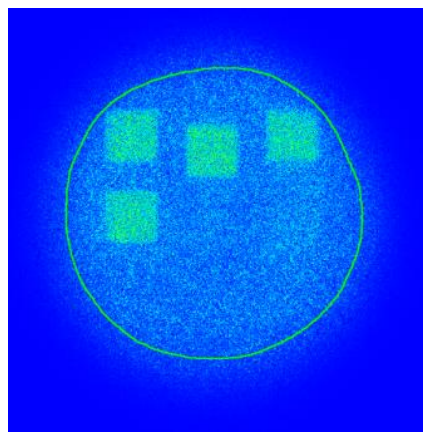

(b) 17296 iteration steps

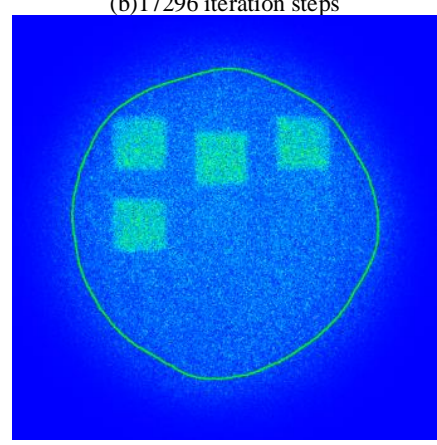

(d) 49680 iteration steps

Figure 9. Time evolution chart of the transverse spatial spot profile of the X-direction polarized laser beam with different iteration steps

\section{CONCLUSION}

In order to support the three-dimensional laser-plasma interaction program, the laser-plasma interaction 3D simulation studies the large-scale filament formation phenomenon under the physical conditions of the black cavity, and analyzes the beam divergence phenomenon and spectral spatial light intensity distribution characteristics after filament formation. Threshold segmentation method for the description of the spatial distribution characteristics of the light spot and the image pixel-based spot contour drawing algorithm realize the visual analysis of the beam divergence mechanism during the propagation of the beam light in the plasma. According to the spot imaging characteristics and random noise characteristics, the outline of the spot propagated by the plasma laser is described. Experimental results show that the algorithm can achieve 
accurate positioning of the spot profile, assist scientific researchers in insight and understanding of key physical issues, improve the accuracy and efficiency of data analysis. This method can realize the precise location of laser spot outline of simple physical model. The disadvantage is that the influence of random disturbance factors on the laser profile is ignored. It is hoped that a laser spot contour algorithm with high robustness and practicability can be designed in combination with practical needs and considering all aspects of random factors.

\section{ACKNOWLEDGEMENT}

This work was supported by National Key R\&D Program of China (No. 2017YFB0202203).

\section{REFERENCES}

Cardema, J. et al., 2008. Performance and characterization results of a lasercom testbed for the pointing, acquisition, and tracking subsystem of a satellite-to-satellite laser communications 1ink. SPIE.

Casey, L. et al., 1995. Laser Communications in Space, Artech House.

Jeppe, F. et al., 2017. On the 'centre of gravity' method for measuring the composition of magnetite/maghemite mixtures, or the stoichiometry of magnetite-maghemite solid solutions, via 57Fe Mössbauer spectroscopy. Journal of Physics D: Applied Physics.

Kurt, O. and Arslan, O., 2012. Geometric interpretation and precision analysis of algebraic ellipse fitting using least squares method. Acta Geodaetica Et Geophysica Hungarica, Vol. 47, No. 4, pp. 430-440.

Li, P. Y., 2013. Research on the center of the sun's position detection algorithm based on image processing. Taiyuan: Taiyuan University of Science and Technology.

Liu, H. L. et al, 2014. An improved laser spot center location algorithm. Computer Measurement and Control, Vol. 22, No. 1, pp. 139-140.

Lu, B. D., 2003. Laser optics: beam description, propagation transformation and cavity technical physics. Higher Education Press, China.

Orujov, F. et al., 2020. Fuzzy based image edge detection algorithm for blood vessel detection in retinal images. Applied Soft Computing Journal.

Qin, Y. et al., 2006. Research on laser spot location of multi circle fitting method. Journal of Xi'an University of Science And Technology, Vol. 26, No. 4, pp. 519-522.

Shen, Y. and Li, G. Y., 2012. Image Threshold Segmentation Method Design. Mechanical Engineering and Automation, Vol. 0, No. 5, pp. 45-46.

Singh, R. et al., 2008. Analysis of accuracy of laser spot centroid estimation. Proceedings of SPIE - The International Society for Optical Engineering.

Tang, G. Q., 2009. Comparison of several laser spot center location algorithm. Journal of Beijing Institute of Mechanical Industry, Vol. 24, No. 1, pp. 62-64

Wang, Y. L. et al., 2007. Laser spot detection and characteristic analysis in space optical communication, Infrared and Laser Engineering, Vol. 36, No. s1, pp. 434-438.

Wang, Z. Q. et al., 2011. Precise positioning algorithm of laser spot center in deformation measurement system. Electronic Measurement and Instrument, Vol. 25, No. 6, pp. 485-486.

Wang, Z. Z. et al., 2013. Center detection algorithm of non full circle laser spot center based on circle fitting. Laser \& Infrared, Vol. 43, No. 6, pp. 709-711.

$\mathrm{Wu}$, D. et al., 2017. Spot center extraction based on cross correlation and improved gaussian fitting. Journal of Optoelectronics Laser, Vol. 28, No. 2, pp. 202-210.

Wu, Y. N. et al., 2011. Investigation on a Scheme for Trajectory Filtering Based on Process Noise Control. Journal of Air Force Engineering University (Natural Science Edition).

Yu, W. L., 2006. Research on the method of calculating the center position of the laser spot. Changchun: Changchun University of Science and Technology.

Zhao, Y. H. el al., 2014. The method of calculating the center of the light spot based on morphology and optical flow equation. Laser \& Infrared, Vol. 44, No. 2, pp. 214-216. 